American Journal of Applied Sciences 6 (4): 691-695, 2009

ISSN 1546-9239

(C) 2009 Science Publications

\title{
Synthesis and Characterization of Gold Nanoparticles by Tryptophane
}

\author{
${ }^{1}$ Azim Akbarzadeh, ${ }^{2,3}$ Davood Zare, ${ }^{1}$ Ali Farhangi, ${ }^{1}$ Mohammad Reza Mehrabi, ${ }^{1}$ Dariush Norouzian, \\ ${ }^{2}$ Shahram Tangestaninejad, ${ }^{2}$ Majid Moghadam and ${ }^{1}$ Nasim Bararpour \\ ${ }^{1}$ Department of Pilot Biotechnology, Pasteur Institute of Iran, Tehran, Iran \\ ${ }^{2}$ Department of Chemistry, Faculty of Science, Isfahan University, Isfahan, Iran \\ ${ }^{3}$ Young Researcher Club, Islamic Azad University, Tehran, Iran
}

\begin{abstract}
Problem statement: Preparation and synthesis of gold nanoparticles with small size and suitable stability is very important and applicable particularly in medicine. In this study, we have prepared gold nanoparticles by chemical reduction method employing L-Tryptophane as a reducing agent for ionic gold. Approach: The gold nanoparticles are the most employed amongst the different metallic nanoparticles in the fields of nanomedicine and nanobiotechnology. Therefore, the employed method should provide suitable particle size, shape and particle distribution in order to obtain nanoparticles of high activity and efficiency indicating the importance of the technique. In this study, $\mathrm{HAuCl}_{4} .3 \mathrm{H}_{2} \mathrm{O}$, L-Tryptophane and polyethyleneglycol (PEG) were used to produce $\mathrm{AuCl}^{-}$ions. They were acted as pre-material, reducing and stabilizing agents respectively. Results: The size, distribution and formation of gold nanoparticles were confirmed by Transmission Electron Microscopy (TEM) indicating the diameter of gold nanoparticles at the range of 10-25 nm and UV spectroscopy. The formed nanoparticles showed the highest absorption at $518 \mathrm{~nm}$. Conclusion: The gold nanoparticles were stable in PEG1000. Since these nanoparticles have suitable size distribution they can be considered as a suitable candidate to be employed in nanomedicine and nanobiotechnology.
\end{abstract}

Key words: Gold nanoparticles, chemical reduction, L-tryptophane, stability

\section{INTRODUCTION}

Metal nanoparticles have been intensively studied within the past decade. Nanosized materials have been an important subject in basic and applied sciences; the unique properties of nanoparticles sparked their application in a broad ranges of different fields, including chemistry, physics, biology, materials science, medicine, catalysis and so on ${ }^{[1-4]}$. Nanoparticles can be used as labels for optical biodetection, substrate for multiplexed aqueous bioassays, probes for cellular imaging or carriers for therapeutic delivery ${ }^{[5-6]}$. Metal nanoparticles are attractive due to their easy synthesis, modification as well as their size, shape, distribution which are properties dependent ${ }^{[7-8]}$. In particular, gold nanoparticles can be synthesized and stabilized by peptides, proteins, DNA and chemical/biological polymers ${ }^{[9-11]}$. Several classes of synthesis methods exist thus displaying different characteristics of the nanoparticles. Basically nanoparticles can be spectroscopically characterized on the basis of their sizes and the method can reveal the concentration of the synthesized nanoparticles too ${ }^{[12-15]}$. Besides, the strength of the reductant and action of the stabilizer in aqueous phase of the system is critical. Different chemicals have the potentials serve as reducing agent in the process of nanoparticles production. These reagents could be either inorganic such as sodium/potassium borohydrate $e^{[16]}$, hydrazine $e^{[17]}$ and salts of tartarate ${ }^{[18]}$, or organic ones like, sodium citrate ${ }^{[19]}$, ascorbic acid ${ }^{[20-21]}$ and amino acids capable of being oxidized ${ }^{[22-23]}$. When the nanoparticles are formed, they need to be stabilized for further use. Various reagents have been reported to serve as stabilizing agent. These include the polymers such as different kind of polyethyleneglycol ${ }^{[24-25]}$, polyvinyl alcohol ${ }^{[26]}$, polyvinyl pyrilidon ${ }^{[27,28]}$ and the surfactant viz, sodium dodycel sulfate ${ }^{[29-31]}$, tween 80 , triton $^{[32]}$ and carbohydrates like chitosane ${ }^{[33]}$.

In this study attempts are made to synthesis gold nanoparticles by chemical reduction technique employing L-Tryptophane as reducing agent. There are reports indicating use of this method synthesize different nanoparticles such as silver, titanium oxide, iron oxide with different reducing/stabilizing agents $^{[34-37]}$.

Corresponding Author: Azim Akbarzadeh, Department of Pilot Biotechnology, Pasteur Institute of Iran No. 358, 12 Farvardin Street, Jomhoori Avenue, Tehran, Iran 13169-43551

Tel: +98 2166465406 Fax: +98 2166465132 


\section{MATERIALS AND METHODS}

Tetrachloro auric acid trihydrate, L-Tryptophane, polyethyleneglycol 1000 were obtained from Merck Company. Deionized water was used through out the experiments.

Synthesis of gold nanoparticles: Solutions of $\mathrm{HAuCl}_{4} \cdot 3 \mathrm{H}_{2} \mathrm{O}$, L-Tryptophane and polyethyleneglycol 1000 at concentration of $2.25 \mathrm{mM}$ and $3.3 \%$ were prepared respectively. Gold nanoparticles were synthesized by Turkevich method as modified by us. In brief, $10 \mathrm{~mL}$ of $\mathrm{HAuCl}_{4} \cdot 3 \mathrm{H}_{2} \mathrm{O}$ was heated to its boiling on a magnetite stirrer, to which, $15 \mathrm{~mL}$ of reducing agent was injected. Heating was continued till the color of the solution changed from colorless to pink/red. Three milliliter of $3.3 \%$ polyethyleneglycol 1000 at room temperature was added to the above mentioned solution.

\section{Identification:}

- UV-Vis spectroscopy: The formation of gold nanoparticles was followed by scanning the solution containing gold nanoparticles at the wave length ranged from 400-700 nm using Shimadzu UV-1601 spectrophotometer

- Transmission Electron Microscopy (TEM): The analysis of synthesized gold nanoparticles was carried out on the film coated drop of nanoparticles employing transmission electron microscopy JEOL, JEN2010

\section{RESULTS}

The formation of gold nanoparticles was followed by measuring the absorption of the solution containing gold nanoparticles at the wave length ranged from 400$700 \mathrm{~nm}$. The maximum absorption was obtained at wave length $518 \mathrm{~nm}$ showing the formation of gold nanoparticles (Fig. 1) ${ }^{[40-42]}$.

The stability of the product is an important criterion in synthesizing nanoparticles that needs to be considered. To stabilize the formed gold nanoparticles, we employed PEG 1000. Figure 2 and 3 reveal the importance of the stabilizer in the system. The formed gold nanoparticles were stable for one month while those without stabilizer were stable for one week. Transmission electron microscopy of synthesized gold nanoparticles shows the diameter of the nanoparticles ranging from $10-25 \mathrm{~nm}$ (Fig. 4). In this way by employing a simple and one step reaction we could synthesize gold nanoparticles in the presence of tryptophane and PEG 1000 as reducing and protecting agents in the size of 10-25 with one month stability promising its application in nanomedicine, nanobiotechnology and other related fields.

\section{DISCUSSION}

Gold nanoparticles can be synthesized by chemical reduction method of Turkevich. But the synthesis of gold nanoparticles by this method requires the reduction of $\mathrm{AuCl}_{4}{ }_{4}$ ions to gold nanoparticles. Furthermore, there are many chemical compound and reagents which can be considered as reducing agents. Kimling et al. ${ }^{[38]}$ used Turckevich method to synthesis gold nanoparticles at the range of $9-120 \mathrm{~nm}$ in the presence of sodium citrate and ascorbic acid whereas Bhargava et al ${ }^{[23]}$ synthesized the gold nanoparticles in the range of 5-15 nm employing different amino acids viz, tyrosine, arginine and mixture of glycine-tyrosine also Joshi et al. ${ }^{[22]}$ synthesized the gold nanoparticles by Lysine; Later on May et al. ${ }^{[39]}$ obtained gold nanoparticles in the rang of $15-25 \mathrm{~nm}$ in the presence of amino-dextran as reducing and protecting agents. Nevertheless, the synthesis of gold nanoparticles has been evolutionized since the time of Turckevich employing aforementioned. While synthesizing gold nanoparticles, reduction of anions is important and this is brought about by tryptophane. Tryptophane is an

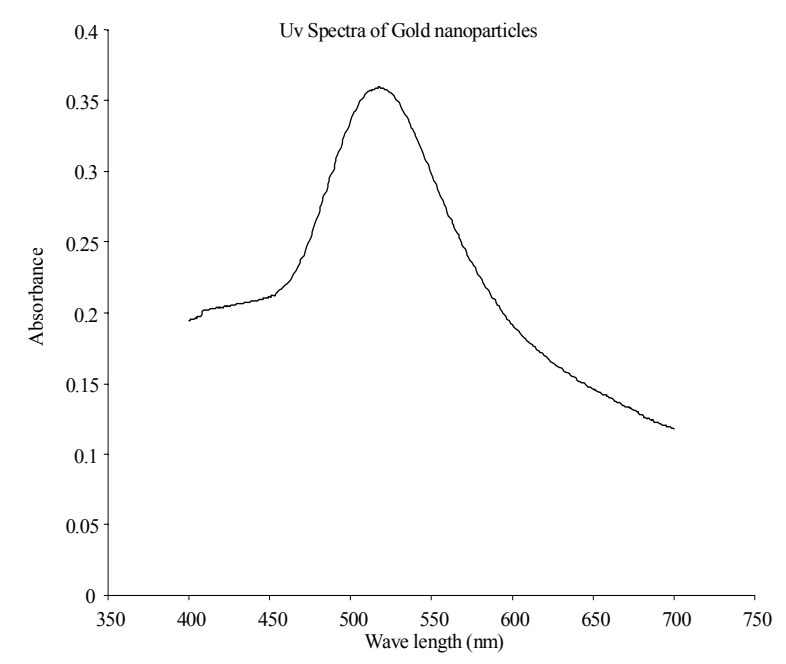

Fig. 1: UV-Vis Spectrum of Gold nanoparticles

amino acid that can be oxidized into its Kynurenine form, thus this amino acid can be used as a reducing agent while preparing gold nanoparticles. The 
mechanism of the reaction is such that the carboxylic group of tryptophane loses electron to be converted to carbonyl and $\mathrm{AuCl}^{-}$gains the electron to be converted to $\mathrm{Au}$ (scheme 1). Nanoparticles are being formed in various sizes, therefore, their absorption spectra vary and the obtained peak depends on the size of the nanoparticles. Wagner et al. ${ }^{[28]}$ Humbert et al. ${ }^{[40]}$ and Kumar Jena et al. ${ }^{[41]}$ synthesized gold nanoparticles in the ranges of 5-50, 20 and $60 \pm 5 \mathrm{~nm}$ showing maximum absorption at $\lambda 527,525$ and $532 \mathrm{~nm}$ respectively revealing that maximum absorption of synthesized gold nanoparticles varies with sizes of the synthesized nanoparticles.

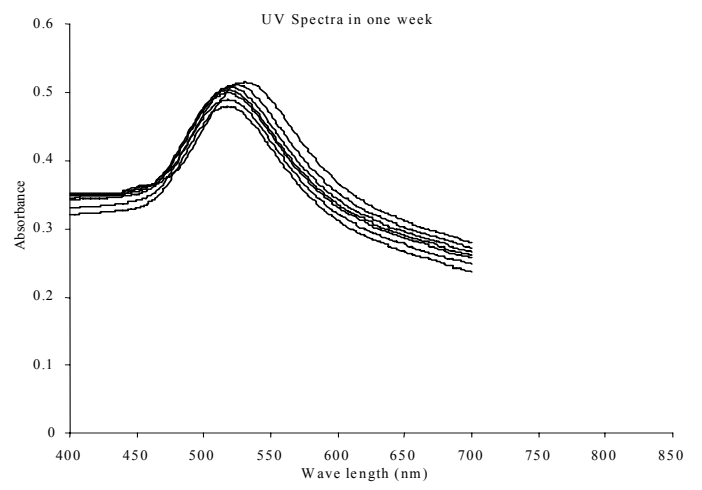

Fig. 2: UV-Vis Spectrum of Gold nanoparticles in one

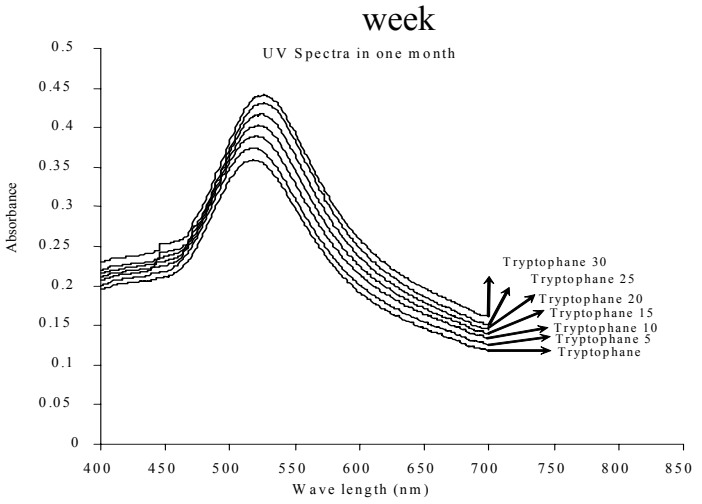

Fig. 3: UV-Vis Spectrum of Gold nanoparticles in one month

Scheme 1- Oxidation mechanism of L-Tryptophane with $\mathrm{HAuCl}_{4}$ and reduction of $\mathrm{AuCl}_{4}^{-}$

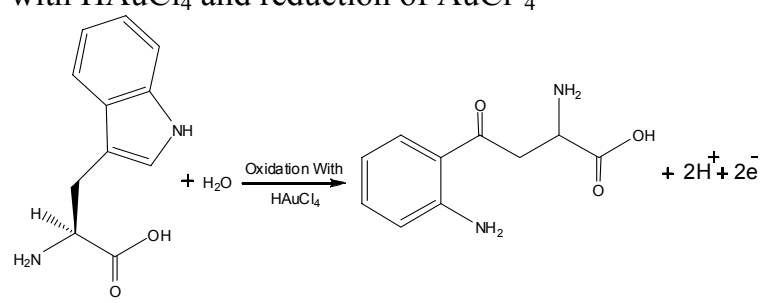

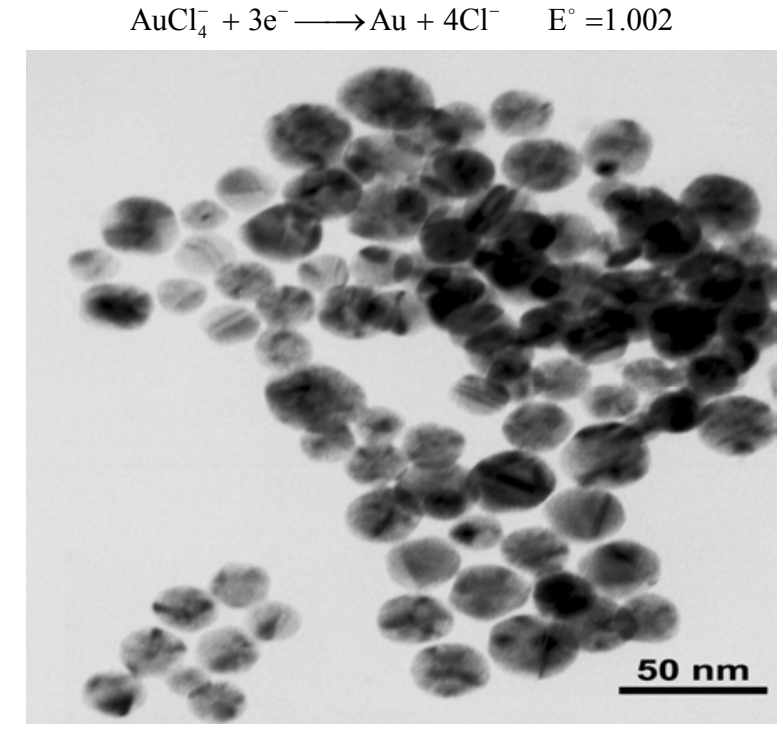

Fig. 4: TEM image of Gold Nanoparticles

\section{REFERENCE}

1. Watanabe, K., D. Menzel, N. Nilius, H.J. Freund, 2006. Photochemistry on metal nanoparticles. Chem. Rev., 106: 4301- 4320. DOI: $10.1021 / \mathrm{cr} 050167 \mathrm{~g}$

2. Salata, O. 2004. Nanoparticles-known and unknown health risks. J. Nanobiotechnol., 2: 1-12. DOI: 10.1186/1477-3155-2-12

3. Mirkin, C.A. and T.A. Taton, 2000. Materials chemistry: Semiconductors meet biology. Nature, 405: 626-627. DOI: 10.1038/35015190

4. Yang, P., W. Zhang, Y. Du and X. Wang, 2006. Hydrogenation of nitrobenzenes catalyzed by platinum nanoparticle core-polyaryl ether trisacetic acid ammonium chloride dendrimer shell nanocomposite. J. Molecular Catalysis A, 260: 4-10. DOI: 10.1016/j.molcata.2006.06.045

5. Wei, H., X. Zhang, C. Cheng, S.X. Cheng and R.X. Zhuo, 2007. Self-assembled, thermosensitive micelles of a star block copolymer based on PMMA and PNIPAAm for controlled drug delivery. Biomaterials, 28: $99 . \quad$ DOI: 10.1016/j.biomaterials.2006.08.030

6. Torchilin, V.P. 2006. Multifunctional nanocarriers. Advanced Drug Delivery Rev., 58: 1532-1555. DOI: 10.1016/j.addr.2006.09.009

7. Burda, C., X. Chen, R. Narayanan and M.A. ElSayed, 2005. Chemistry and properties of nanocrystals of different shapes. Chem. Rev., 105: 1025-1102. DOI: $10.1021 / \mathrm{cr} 030063 \mathrm{a}$ 
8. Cushing, B.L., V.L. Kolesnichenko and C.J. O'Connor, 2004. Recent Advances in the liquid-phase syntheses of inorganic nanoparticles. Chem. Rev., 104: 3893-3946.

DOI: $10.1021 / \mathrm{cr} 030027 \mathrm{~b}$

9. Seeman, N.C., 2003. Biochemistry and structural DNA Nanotechnology: An evolving symbiotic relationship. Biochemistry, 42: 7259-7269.

DOI: $10.1021 / \mathrm{bi030079v}$

10. Vijayanathan, V., T. Thomas, T.J. Thomas, 2002. DNA nanoparticles and development of DNA delivery vehicles for gene therapy. Biochemistry, 41: 14085-14094. DOI: 10.1021/bi0203987

11. Okugaichi, A., K. Torigoe, T. Yoshimura and K. Esumi, 2006. Interaction of cationic gold nanoparticles and carboxylate-terminated poly (amidoamine) dendrimers. Colloids Surfaces A., 273: 154-160. DOI: 10.1016/j.colsurfa.2005.08.028

12. Brust, M., M. Walker, D. Bethell, D.J. Schiffrin and R.J. Whyman, 1994. Synthesis of thiolderivatised gold nanoparticles in a two-phase Liquid-Liquid system. Chem. Soc., Chem. Commun., 801-802. DOI: 10.1039/C39940000801

13. Turkevich, J., P.C. Stevenson and J. Hillier, 1951. Preparation of $2.5 \times 10^{-4} \mathrm{M}$ gold colloids (Sodium citrate reduction method). Discuss. Faraday Soc., 11: 55. DOI: 10.1039/DF9511100055

14. Turkevich, 1985. Historical and preparative aspects, morphology and structure. J. Gold Bull., 18:86.

(URL:http://www.goldbulletin.org/downloads/Turk evich_3_18.pdf)

15. Frens, G., 1973. Controlled nucleation for the regulation of the particle size in monodispersed gold suspensions. Nat. Phys. Sci., 20: 241.

16. Walker, C.H., J.V.St. John and P. Wisian-Neilson, 2001. Synthesis and size control of gold nanoparticles stabilized by poly (methylphenylphosphazene). J. Am. Chem. Soc., 123: 3846. DOI: $10.1021 / \mathrm{ja} 005812+$

17. Kawasaki, H., K. Nishimura, R. Arakawa, 2007. Influence of the counterions of cetyltrimetylammonium salts on the surfactant adsorption onto gold surfaces and the formation of gold nanoparticles. J. Phys. Chem. C., 111: 2683-2690. DOI: 10.1021/jp066963n

18. Das, R.N. and P. Pramanik, 2000. Chemical synthesis of fine powder of lead magnesium niobate using niobium tartarate complex. Mater.
Lett., 46: 7. DOI: 10.1016/S0167-577X(00)001348

19. Schulz-Dobrick, M., K.V. Sarathy and M. Jansen, 2005. Surfactant-free synthesis and functionalization of gold nanoparticles. J. Am. Chem. Soc., 127: 12816. DOI: 10.1021/ja054734t

20. Vemula, P.K., U. Aslam, V.A. Mallia and G. John, 2007. In situ synthesis of gold nanoparticles using molecular gels and liquid crystals from vitamin-C amphiphiles. Chem. Mater., 19: 138. DOI: $10.1021 / \mathrm{cm} 062464 \mathrm{n}$

21. Jana, N.R., L. Gearheart and C.J. Murphy, 2001. Wet chemical synthesis of high aspect ratio cylindrical gold nanorods. J. Phys. Chem. B., 105: 4065. DOI: 10.1021/jp0107964

22. Joshi, H., P.S. Shirude, V. Bansal, K.N. Ganesh and M. Murali Sastry, 2004. Isothermal titration calorimetry studies on the binding of amino acids to gold nanoparticles. J. Phys. Chem. B., 108: 11535. DOI: 10.1021/jp048766z

23. Bhargava, S.K., J.M. Booth, S. Agrawal, P. Coloe and G. Kar, 2005. Gold nanoparticle formation during bromoaurate reduction by amino acids. Langmuir, 21, 5949. DOI: 10.1021/la050283e

24. Haba, Y., C. Kojima, A. Harada, T. Ura, H. Horinaka and K. Kono, 2007. Preparation of poly (ethylene glycol)-modified poly (amido amine) dendrimers encapsulating gold nanoparticles and their heat-generating ability. Langmuir, 23: 5243. DOI: 10.1021/la0700826

25. Bodnar, M., J.F. Hartmann and J. Borbely, 2006. Synthesis and study of cross-linked chitosan-Npoly (ethylene glycol) nanoparticles. Biomacromolecules, 7: 3030 . DOI: 10.1021/bm0605053

26. Tripathy, P., A. Mishra and S. Ram, 2007. Immobilizing Au-nanocolloids in co-branched polymer molecules in presence of gluconic acid in poly (vinyl alcohol) in hot water. Mater. Chem. Phys., 106 : $379 . \quad$ DOI: 10.1016/j.matchemphys.2007.06.016

27. Salvati, R., A. Longo, G. Carotenuto, S. De Nicola, G.P. Pepe, L. Nicolais and A. Barone, 2005. UVvis spectroscopy for on-line monitoring of $\mathrm{Au}$ nanoparticles size during growth. Applied Surface Sci., 248 : 28-31. 10.1016/j.apsusc. 2005.03 .075

28. Wagner, J. and J.M. Kohler, Continuous synthesis of gold nanoparticles in a micro reactor. Nano Lett., 5: 685-691. DOI: 10.1021/n1050097t 
29. Mafune, F., J. Kohno, Y. Takeda and T. Kondow, 2001. Formation of gold nanoparticles by laser ablation in aqueous solution of surfactant. J. Phys. Chem. B., 105: 5114. DOI: 10.1021/jp0037091

30. Kuo, C.H., T.F. Chiang, L.J. Chen and M.H. Huang, 2004. Synthesis of highly faceted pentagonal- and hexagonal-shaped gold nanoparticles with controlled sizes by sodium dodecyl sulfate. Langmuir, 20: 7820. DOI: 10.1021/la049172q

31. Deng, J.P., C. Wu, G.H. Yang and C.Y. Mou, 2005. Pyrene-assisted synthesis of size-controlled gold nanoparticles in sodium dodecyl sulfate micelles. Langmuir, 21: 8947. DOI: 10.1021/la051252m

32. Lu, C., Y. Zu and V.W.W. Yam, 2007. Nonionic surfactant-capped gold nanoparticles as postcolumn reagents for high-performance liquid chromatography assay of low-molecular-mass biothiols. J. Chromatograp. A, 1163: 328. DOI: 10.1016/j.chroma.2007.07.045

33. Huang, H. and X. Yang, 2004. Synthesis of chitosan-stabilized gold nanoparticles in the absence/presence of tripolyphosphate. Biomacromolecules, 5: 2340 . DOI: 10.1021/bm0497116

34. Xu, C. and A.S. Teja, 2008. Continuous hydrothermal synthesis of iron oxide and PVAprotected iron oxide nanoparticles. J. Supercritical Fluids, 44: 85. DOI: 10.1016/j.supflu.2007.09.033

35. Daou, T.J., G. Pourroy, S. Begin-Colin, J.M. Greneche, C. Ulhaq-Bouillet, P. Legare, P. Bernhardt, C. Leuvrey and G. Rogez, 2006. Hydrothermal synthesis of monodisperse magnetite nanoparticles. Chem. Mater, 18: 4399. DOI: $10.1021 / \mathrm{cm} 060805 \mathrm{r}$
36. Jeon, S. and P.V. Braun, 2003. Hydrothermal synthesis of Er-doped luminescent $\mathrm{TiO}_{2}$ nanoparticles. Chem. Mater., 15: 1256. DOI: $10.1021 / \mathrm{cm} 0207402$

37. Yu, D. and V.W.W. Yam, 2005. Hydrothermalinduced assembly of colloidal silver spheres into various nanoparticles on the basis of HTABmodified silver mirror reaction. J. Phys. Chem. B., 109: 5497. DOI: 10.1021/jp0448346

38. Kimling, J., M. Maier, B. Okenve, V. Kotaidis, H. Ballot and A. Plech, 2006. Turkevich method for gold nanoparticle synthesis revisited. J. Phys. Chem. B., 110: 15700. DOI: 10.1021/jp061667w

39. Ma, Y., N. Li, C. Yang and X. Yang, 2005. Onestep synthesis of amino-dextran-protected gold and silver nanoparticles and its application in biosensors. Anal. Bioanal. Chem., 382: 1044. DOI: 10.1007/s00216-005-3222-4

40. Humbert, C., B. Busson, J.P. Abid, C. Six, H.H. Girault and A. Tadjeddine, 2005. Self-assembled organic monolayers on gold nanoparticles: A study by sum-frequency generation combined with UVvis spectroscopy. Electrochemical Acta, 50: 3101. DOI:10.1016/j.electacta.2004.10.084

41. Kumar Jena, B., C.R. Raj, 2007. Synthesis of flower-like gold nanoparticles and their electrocatalytic activity towards the oxidation of methanol and the reduction of oxygen. Langmuir, 23: 4064. DOI: 10.1021/la063243z

42. Sardar, R., J.W. Park, J.S. Shumaker-Parry, 2007. Polymer-induced synthesis of stable gold and silver nanoparticles and subsequent ligand exchange in water. Langmuir, 23: 11883 . DOI: $10.1021 / 1 \mathrm{la} 702359 \mathrm{~g}$ 